\section{RELATION BETWEEN SERUM BONE TURNOVER MARKERS, ESTRADIOL, AND PHYSICAL DEVELOPMENT IN HEALTHY GIRLS OF PUBERTAL AGE}

B. Kulik-Rechberger ${ }^{1}$, B. Mozejko-Pastewka², W. Furmaga-Jablonska ${ }^{3}$

${ }^{1}$ Department of Paediatric Propedeutics, Medical University, Lublin, ${ }^{2}$ Eli Lilly, Area Medical Center Vienna, Warszawa, 'Department of Neonates' and Infants' Pathology, Medical University, Lublin, Poland

Biochemical measurements of bone turnover parameters are helpful in the study of the pathophysiology of skeletal metabolism and growth. However interpretation of their results is still not clear in children in relation to different ages and gender.

Aim:Toassesstherelationshipofserumconcentration of bone turnover markers: osteoprotegerin (OPG), receptor activator of nuclear factor (NF)-KB ligand (sRANKL), procollagen type I N-terminal propeptide (PINP) and cross-links of type I collagen (sCTX) to estradiol, age of menarche, and anthropometric parameters of pubertal girls.

Methods: The study was performed on 92 healthy girls (age: 11,8-13,7y.) divided into two subgroups depending on menarche status (after and before menarche).

Results: No correlation was found between OPG and SRANKL vs other biochemical parameters (PINP, sCTX, estradiol) as well as physical parameters for the whole group. We have found positive correlation between PINP and SCTX ( $r=0,4294, p<0,0001)$. There was negative correlation between estradiol and PINP as well sCTX ( $r=-0,3122, p=0,005$ and $r=-$ $0,2409, p=0,031$ respectively). Also PINP and $s C T X$ but not OPG, and SRANKL correlated negatively with menarche status, body weight, BMI, bi-iliac width and positively with height, and leg length increases. Mean concentrations of OPG and SRANKL were similar in both subgroups. There was significant difference in mean serum concentrations of estradiol after/before menarche groups $(51,6 \pm 18,8 \mathrm{pg} / \mathrm{mL}$, and $27,5 \pm 15$ $\mathrm{pg} / \mathrm{mL}$ respectively), PINP $(549,3 \pm 255,2 \mu \mathrm{g} / \mathrm{L}$, and $864,5 \pm 392,5 \mu \mathrm{g} / \mathrm{L}$ respectively), sCTX $(2,1 \pm 0,6 \mathrm{ng} /$ $\mathrm{mL}$, and $2,8 \pm 0,7 \mathrm{ng} / \mathrm{mL}$ respectively).

Conclusion: In healthy girls in puberty PINP and sCTX but not sRANKL and OPG correlate with menarche status, estradiol concentration and physical development.

\section{9}

\section{HOME BASED CHILD CARE FOR REDUCING CHILD MALNUTRITION IN MELGHAT}

\author{
K.A. Satav \\ Ophthalmology, MAHAN-Mahatma Gandhi Tribal \\ Hospital, Karmagram, Amaravati, India
}

Background: Melghat is tribal area in India with very high child mortality \& malnutrition. One of the major causes of mortality \& morbidity are protein energy malnutrition(PEM). We developed Home Based Child Care (HBCC) model for tribal population to reduce children mortality and malnutrition.

\section{Objectives:}

1. To reduce Infant Mortality rate (IMR) \& Under 5 children mortality rate(U5MR) (especially due to malnutrition) from $90, \& 140$ to $58.05 \& 72.1$ per 1000 live births respectively in population of 14,120 of Melghat over 3 years.

2. To reduce incidence of severe malnutrition in above population by $35 \%$ over 3 years.

Methods: Study-design was Randomised Control Trial. We selected 16 intervention (population 14,888 ) and 18 control (population 16,310) villages. Trained village health workers in intervention area treated post-neonatal diseases such as malnutrition, diarrhoea, ARI, malaria and neonatal diseases like LBW, etc. Behaviour Change Communication programs were conducted.

Results: Baseline mortality indices and prevalence of severe malnutrition in control versus intervention areas were: IMR- 72.97 vs 94.9, \& U5MR- 102.56 vs 143.52 and severe malnutrition- $9.6 \%$ vs.9.8 \%. After intervention IMR, U5MR \& prevalence of severe malnutrition were significantly decreased in intervention area to $24.79,37.19 \& 4.4 \%$ respectively $(p<0.05)$. The prevalence, number of deaths \& case fatality rates due to malnutrition in intervention area were reduced significantly $(p<$ 0.05).

Conclusions: $\mathrm{HBCC}$ resulted in significant decrease in children mortality especially due to malnutrition \& prevalence of severe malnutrition. Our model is replicable for reducing children mortality due to malnutrition. 